\title{
Studies of Bimaterial Interface Fracture with Peridynamics
}

\author{
Fang Wang ${ }^{1}$, Lisheng Liư ${ }^{2, \star}$, Qiwen Liư ${ }^{1}$, Zhenyu Zhang ${ }^{1}$, Lin Su ${ }^{1} \&$ Dan Xue ${ }^{1}$ \\ ${ }^{1}$ Department of Engineering Structure and Mechanics, Wuhan University of Technology, Wuhan, \\ 430070, China \\ ${ }^{2}$ State Key Laboratory of Materials Synthesis and Mechanics, Wuhan University of Technology, \\ Wuhan, 430070, China
}

Keywords: Peridynamic theory, bimaterial interface, dynamic fracture, nonlocal methods.

\begin{abstract}
The peridynamic is a kind of unique theory which can deal with continuous models, defect of crack and particles in a single framework. It does this by formulating problems in terms of integro-differential equation rather than partial differential equations. This theory can be used to investigate the dynamic mechanical behavior of the discontinuous material, such as the propagation, bifurcation, unstability process of crack. However, there is no reasonable force function for the interface of bimaterial. In this paper, we utilize a weight function method to establish the force function of the bimaterial interface. We discussed crack propagation along the interface in a prenotch bimaterial model by peridynamics, and analyzed the influence of different mesh shapes on the deflection angle of crack path. By comparing the peridynamic solution for these problems with the results published in the literature, we concluded that peridynamics with the proposed method is a reliable formulation for modeling the interfacial dynamic fracture of bimaterial.
\end{abstract}

\section{Introduction}

Fracture along or near a bimaterial interface plays a central role in limiting the toughness of composite material. This has motivated a substantial body of work on failure at bimaterial interface. The fracture mechanics for bimaterial interface crack growth has been developed several decades. The identification of crack tip parameters of bimaterial interfacial cracks is much complex than for cracks in homogeneous solids because of highly non-linear in stress field and material properties.

The problem of elastic bimaterial structure depends on Dundurs parameter. The Dundurs parameter can indicate the modulus matching at vertical and parallel of interface [1]. Based on the classical theory of fracture mechanics, the existing literature generally adopt the plural form stress intensity factor (SIF) defined by Hutchinson et al. The plural form SIF can simplify the problem of bimaterial interface fracture, as it make the energy release rate formula remain consistent with the formula of homogeneous material [2]. However, the theoretical calculation was limited due to the complexity of actual composite material.

In recent years, the numerical method has great development in solving bimaterial interface fracture. Finite element method (FEM) is a sophistical approach to cope with bimaterial interface fracture. Extended finite element method (XFEM) and Cohesive-zone FEM-based methods which are based on the FEM, improve the calculation accuracy and efficiency. XFEM overcomes the shortcoming while retains the advantage of FEM. XFEM which is based on an idea of Partition of Unity, introduces a special function to solve the discontinuous problem. It can improve the computational efficiency without refining the local mesh and remeshing the region near crack tip [3]. The CZM is based on the elastic-plastic fracture mechanics. It is able to avoid yielding on a large scale near crack tip with the idea that stress depend nonlinearly on crack tip opening displacement [1]. Generally, researchers allocate Cohesive-zone elements on known crack path or redistribute the Cohesive-zone elements with the crack growth.

The peridynamic theory is a new way to solve discontinues problem. It has many successful cases of solving dynamic fracture in homogenous material. Initially, Silling and Bobaru [4] used the peridynamic method to simulate the stretching and dynamic tearing of membranes. Silling and Weckner [5] discussed the condition for crack nucleation. Ha and Bobaru [6] applied this method in dynamic crack propagation and crack branching numerical simulations. They had analyzed the 
influence of convergence methods and micro-modulus shapes on crack propagation. Bobaru [7] analyzed the effect of Vander Waals forces on nonhomogeneous nanofiber network mechanical behavior with the peridynamic method, while the peridynamic considering all interactions as 'longrage'.

There are no appropriate method to handle dynamic fracture along the bimaterial interface in peridynamics. In this paper, we utilize a weight function method to establish the force function of the bimaterial interface. The focus in this investigation is on the evolution of the crack path and crack tip stress. Calculations are carried out for a PMMA/Al bimaterial plate. This model has been analyzed by Xu and Needleman [8] by Cohesive-zone FEM-based methods.

\section{The peridynamic formulation for bimaterial interface}

Overview of the peridynamic theory. The peridynamic theory is a kind of nonlocal theory that formulates problem with the integro-differential equation. It integrates the force acting on a material point in a region with finite Horizon [9]. The peridynamic equation of motion is formulated as

$$
\rho \frac{\partial^{2} \mathbf{u}(\mathbf{x}, t)}{\partial t^{2}}=\int_{H} \mathbf{f}\left(\mathbf{u}\left(\mathbf{x}^{\prime}, t\right)-\mathbf{u}(\mathbf{x}, t), \mathbf{x}^{\prime}-\mathbf{x}\right) d V_{x}+\mathbf{b}(\mathbf{x}, t)
$$

where $\rho$ is the density of the particle at $\mathbf{x}, H$ a neighborhood of the particle at $\mathbf{x}$ with a horizon symbolized by $\delta$, $\mathbf{f}$ the pairwise force function in the peridynamic bond that connects the particle at $\mathbf{x}^{\prime}$ to $\mathbf{x}, \mathbf{u}$ is the displacement vector field and $\mathbf{b}(\mathbf{x}, t)$ the prescribed body force density field (Fig. 1).Then we simplify the function by introducing two variables: the relative position in the reference configuration $\xi$ :

and the relative displacement $\boldsymbol{\eta}$ :

$$
\xi=x^{\prime}-\mathbf{x}
$$

$$
\boldsymbol{\eta}=\mathbf{u}\left(\mathbf{x}^{\prime}, t\right)-\mathbf{u}(\mathbf{x}, t)
$$

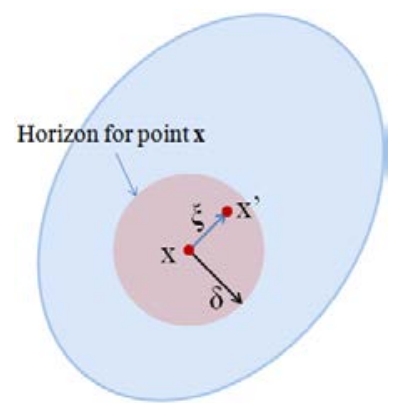

Fig.1: Interaction of a material point with its neighboring points

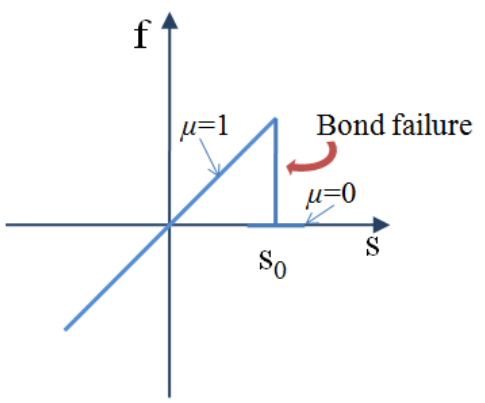

Fig.2: Bond force function with failure

A micro-potential $\omega$ is defined as the energy in a single bond of micro-elastic material. The pairwise force function is defined as:

$$
\mathbf{f}(\boldsymbol{\eta}, \boldsymbol{\xi})=\frac{\partial \omega(\boldsymbol{\eta}, \boldsymbol{\xi})}{\partial \boldsymbol{\eta}}
$$

We can consider the interaction between two particles as an elastic or nonlinear spring. At present, most researchers think that the spring properties depend on the relative position vector $\xi$, and defined a correlation between the bond force and stretch. Silling and Askari [10] defined the pairwise force function for prototype micro-elastic brittle (PMB) material (Fig. 2):

$$
\mathbf{f}(\boldsymbol{\eta}, \boldsymbol{\xi})=\frac{\xi+\boldsymbol{\eta}}{|\xi+\eta|} \operatorname{cs} \mu(\xi, t)
$$

where $c$ is the micro-modulus which can be obtained by bulk modulus $k$ and horizon $\delta$. For twodimensional plate (with thickness $h$ ), the micro-modulus of bond-based peridynamics is: 


$$
c=\frac{12 k}{\pi h \delta^{3}}
$$

and $s$ is the bond stretch:

$$
s=\frac{|\xi+\eta|-|\xi|}{|\xi|}
$$

and $\mu$ is a history-dependent scalar-valued function:

$$
\mu=\left\{\begin{array}{l}
1 \text { if } s\left(\xi, t^{\prime}\right)<s_{0} \text { for all } 0 \leq t^{\prime} \leq t, \\
0 \text { otherwise. }
\end{array}\right.
$$

In addition, the micro-modulus has two shapes: constant and conical. Ha and Bobaru [6] has proved that the two shapes of micro-modulus has little influence on the difference of results. The crack propagation directions are different but similar, and the crack propagation speeds are very close to each other.

The critical stretch $s_{0}$ indicates material damage. The bond between the interacting particles break up, when the bond stretch reaches the critical stretch. This condition is a non-recoverable damage, so the bond force sustains in zero. The critical stretch $s_{0}$ for the PMB material can be obtained by the fracture energy $G_{0}$.

$$
s_{0}=\sqrt{\frac{5 G_{0}}{9 k \delta}}
$$

For some real materials, the critical stretch is not a constant. It may obtained by experiment.

The force function for bimaterial interface. The bond force between different materials is the key point of bimaterial interface fracture numerical simulations with peridynamic theory. The force function of bond-based peridynamic model is based on the bond stretch and the material's micromodulus which is related to the elastic modulus of classical continuum mechanics. The micromodulus for particles with different materials on each side of the interface is an unknown parameter.

In this paper, we develop the mathematical model based on a weight function method. For the ideal interface, the micro-modulus $c$ in the force function of particles made by material A and B can be formulated as:

$$
c=\omega_{A} c_{A}+\omega_{B} c_{B}
$$

Here we define $\omega_{A}=l_{A} / l$ and $\omega_{B}=l_{B} / l$. Then, applying Eq. (5) leads to:

$$
\mathbf{f}(\boldsymbol{\eta}, \boldsymbol{\xi})=\frac{\boldsymbol{\xi}+\boldsymbol{\eta}}{|\xi+\eta|}\left(\omega_{A} c_{A}+\omega_{B} C_{B}\right) s \mu(\xi, t)
$$

The weight function can briefly show the dissimilarity and transitivity of bimaterial interface material. There exist a relationship between the thickness of interfacial transition zone $h$ and the horizon $\delta: h=2 \delta$. Therefore, the grid spacing and horizon in the interfacial transition zone should fulfill the above requirement.

\section{The numerical simulation}

Problem description.For We consider the following setup as a benchmark problem for analyzing the crack propagation phenomena: a pre-notched thin rectangular bimaterial plate, with a pre-notch starting from the top vertical edge of the plate, situated along the bimaterial interface of the plate, as show in Fig.4. The simulation for this model is $2 \mathrm{D}$ simulation. The tension load velocity was $\mathrm{v}$. The specimen materials chosen for this study are PMMA/aluminum.

The material properties for the PMMA are given as an elastic modulus of $E=3.24 \mathrm{Gpa}$, mass density of $\rho=1190 \mathrm{~kg} / \mathrm{m}^{3}$, and Poisson's ration of $v=0.35$. The material properties for aluminum are given as an elastic modulus of $E=80.0 \mathrm{Gpa}$, mass density of $\rho=2700 \mathrm{~kg} / \mathrm{m}^{3}$, and Poisson's ration of $v=0.33$.

The analysis of crack path. The geometric of specimen specified with $\mathrm{W}=20 \mathrm{~mm}, \mathrm{H}=50 \mathrm{~mm}$, $\mathrm{A}=10 \mathrm{~mm}$, with $6 \mathrm{~mm}$ thickness. In the following parts, we divided the point arrangement pattern into hexagon particles (Fig.4 (a)) and quadrilateral particles (Fig.4 (b)), respectively. The peridynamic models consists of 6,000 particles, with the horizon $\delta=2.5 \mathrm{~mm}$. It needs to cut out the 
bond connecting particles on both sides of the pre-notch, simulating non-interaction on both sides of the crack. As in Fig.5, the bonds designated by black lines should cut out in the simulation. The above two models are used to study the crack deflecting and branching behavior with high speed load $300 \mathrm{~m} / \mathrm{s}$.

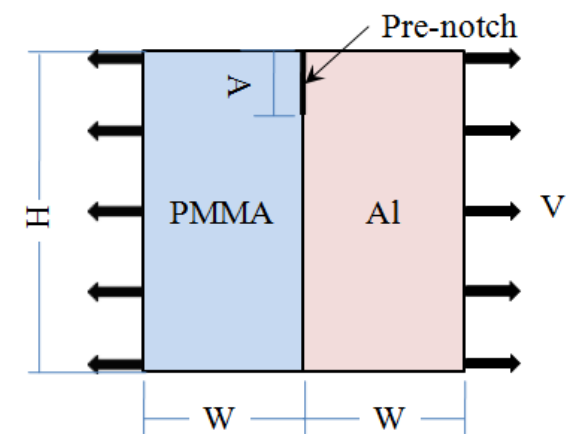

Fig. 3: Schematic plot of specimen with pre-notch

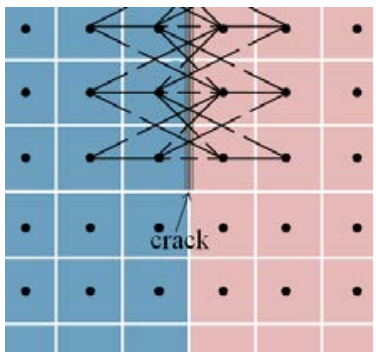

(a)

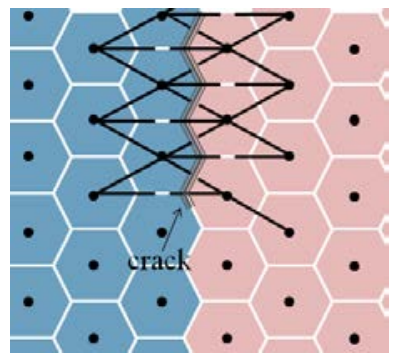

(b)

Fig.4: the numerical simulation model with different point arrangement patterns

The simulation using two different point arrangement patterns instances in Fig.5. The crack growth orientations simulated with the two point arrangement pattern styles are similar. But the deflection angle and the bifurcation position is different. The deflection angle calculated with hexagon point arrangement pattern is larger than the quadrilateral one, due to the shape feature of hexagon.

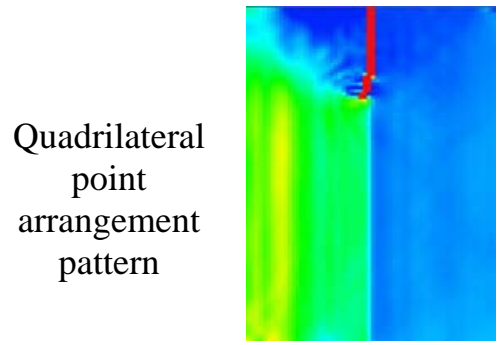

(a)

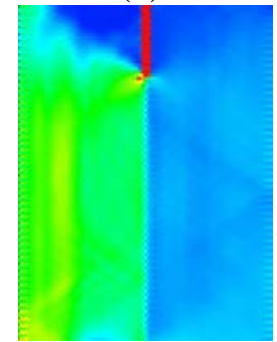

(d)

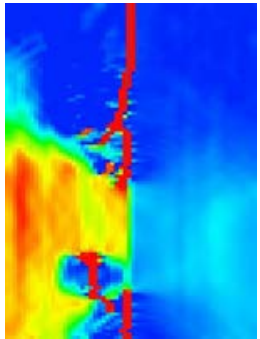

(b)

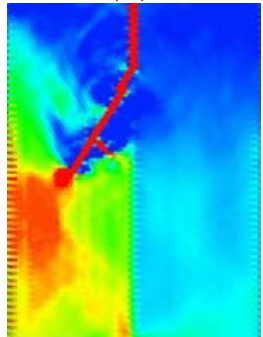

(e)

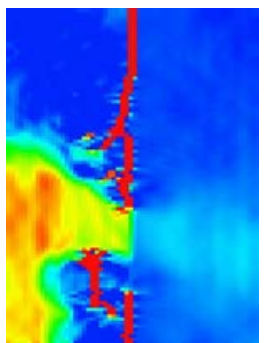

(c)

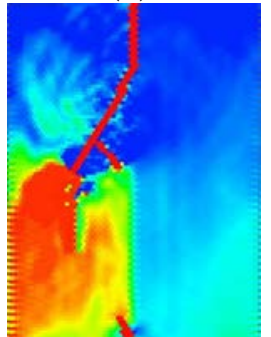

(f)

$$
\mathrm{t}=0.033 \mu \mathrm{s} \quad \mathrm{t}=0.057 \mu \mathrm{s} \quad \mathrm{t}=0.06 \mu \mathrm{s}
$$

Fig.5: the effect of point arrangement pattern on crack growth

The time of the crack initiation simulated by quadrilateral point arrangement pattern is earlier than the hexagon point arrangement pattern (Fig.5 (a) (d)). The crack branching position simulated 
by quadrilateral point arrangement pattern is closer to the initial crack tip position than the hexagon one (Fig.5 (b) (e)). It tends to generate a new crack at the interface of opposite side when the crack progress to a certain extent (Fig.5 (b) (f)). Further analysis, the crack speed in quadrilateral mesh model is faster. As the crack path is close to the interface whose Rayleigh wave is faster.

In order to prove the weight function method's rationality. We have calculated the speed of cracks propagated along the interface between PMMA and aluminum with boundary loading velocity $15 \mathrm{~m} / \mathrm{s}$ (Fig.6).

a) CZM: calculated with cohesive zone model (refer to X.P. Xu and Needleman, Numerical simulations of dynamic crack growth along an interface);

b) $\mathrm{PD}(\mathrm{Hex}$.): calculated by peridynamics with Hexagon point arrangement pattern;

c) $\mathrm{PD}(\mathrm{Qua}$.$) : calculated by peridynamics with quadrilateral point arrangement pattern.$

The crack speed calculated by PD fluctuate around the result curve by Xu \& Needleman.

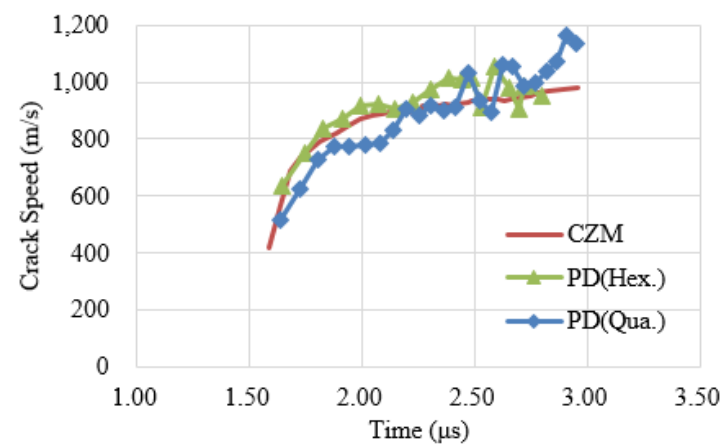

Fig.6: The speed of cracks propagated along the interface

The analysis of crack tip stress. In the following, we add a displacement constraint to model a bimaterial plate with center crack. The geometric of specimen specified with $\mathrm{W}=1.4 \mathrm{~mm}, \mathrm{H}=3 \mathrm{~mm}$, $\mathrm{A}=0.25 \mathrm{~mm}$, with $0.36 \mathrm{~mm}$ thickness. The peridynamic model consists of 6,000 particles (grid spacing is $1 \mathrm{~mm}$ ), with the horizon $\delta=2.5 \mathrm{~mm}$. The contours of normal stress near the crack tip (while the crack length is $0.55 \mathrm{~W}$ ) is show in Fig.7.

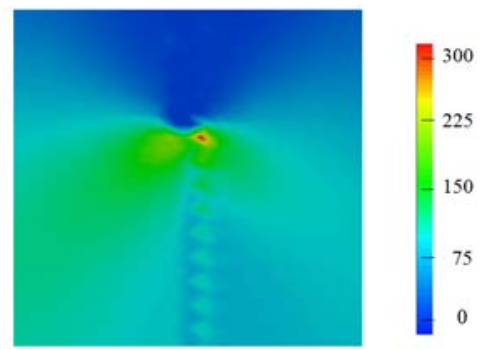

Fig.7: contours of normal stress near the crack tip

The stress contours clearly shows the stress concentration region near the crack tip, and the stress concentration region is asymmetry. The high stress area on the left side made by PMMA is larger than the right. The stress contours feature mentioned above is similar to the result simulated by $\mathrm{Xu}$ and Needleman [8]. But there exists serious stress concentrates on the right side. The maximum value of normal stress is different from these results.

\section{Conclusions}

In this paper, a weight function method has been proposed to describe the bimaterial interface properties. The relationship between the thickness of interfacial transition zone $h$ and the horizon $\delta$ has been determined. This work has examined the capability of the weight function peridynamicbased method to predict crack growth in bimaterial structure. The crack path simulation is sensitive to mesh discretization pattern. It leads to difference in crack speed because of the difference in 
crack speed of dissimilar materials. The stress concentrate emergence near the crack tip, and the stress concentration region is asymmetry because of the difference in dissimilar material properties.

\section{Acknowledgements}

This work is financially supported by the Independent Innovation Research Funds Project (WUT: 2014-zy-171). The corresponding author is Lisheng Liu.

\section{References}

[1] Dundurs, J. Discussion: Edge-bonded dissimilar orthogonal elastic wedges under normal \& shear loading. J. Appl. Mech. 35, 460-466, 1969.

[2] Malyshev, B.M. \& Salganik, R.L. The strength of adhesive joints using the theory of cracks, Int. J. Fracture, 1, 114-128, 1965.

[3] D. B. P. Huynh, T. Belytschko, The extended finite element method for fracture in composite materials, International Journal for Numerical Methods in Engineering, 77, 214-239, 2009.

[4] S. A. Silling \& F. Bobaru, Peridynamic modeling of membranes and fibers, International Journal of Non-Linear Mechanics, 40, 395-409, 2005.

[5] S. A. Silling, O. Weckner, E. Askari, \& F. Bobaru, Crack nucleation in a peridynamic solid, International Journal of Fracture, Vol. 162(1-2), (2010), 219-227.

[6] YD. Ha \& F. Bobaru, Studies of dynamic crack propagation and crack branching with peridynamics, International Journal of Fracture, 162(1-2), 229-244, 2010.

[7] F. Bobaru, Influence of van der Waals forces on increasing the strength and toughness in dynamic fracture of nanofibre networks: a peridynamic approach, Modelling and Simulation in Materials Science and Engineering, 15, 397-417, 2007.

[8] X.-R Xu, A. Needleman, Numerical simulations of dynamic crack growth along an interface, International Journal of Fracture, 74, 289-324,1996.

[9] S. A. Silling \& E. Askari, A meshfree method based on the peridynamic model of solid mechanics, Computers and Structures, 83, 1526-1535, 2005.

[10] S.A. Silling, R.B. Lehoucq, Peridynamic Theory of solid mechanics, Advances in Applied Mechanics, 44, 73-168, 2010. 